\title{
A Study on the Educators' Basic Responsibilities of Traceless Strengthening Educational Activity based on Self-directed Action Mode
}

\author{
Zhe Zhang \\ Wuhan Railway Vocational College of Technology, Wuhan, China
}

\begin{abstract}
As directors of a traceless strengthening educational activity based on self-directed mode, the educators control the whole activity, and hence, it would be not insignificance to study the basic responsibilities of them. As viewed from managing traceless strengthening educational activities based on self-directed mode, the basic responsibilities of the educators are studied in this paper. Firstly, the connotations of traceless strengthening educational activity, self-directed action mode and self-directed team are described. Secondly, according to the requirements of managing the activities, the basic responsibilities of the educators are divided into six aspects. And, the six aspects, one by one, are discussed after the six aspects are defined in detail. And finally, the meaning of the good educators and the general features of a traceless strengthening educational activity are revealed in detail.
\end{abstract}

Index Terms - Traceless strengthening education, self-directed action mode, basic responsibilities, educators, students.

\section{Introduction}

It's well known that the contemporary college students live in a time quite different from the last century when college students have much more opportunities of experiencing and participating in social activities. To be specific, as for the college students in that time, their political ideology and selfreliance played an important role while their desire for materials and wealth was not so strong. People in the last century could always experience the strengthening effect from all aspects of life and cultivate their abilities in self-reliance, and opportunities for receiving the strengthening education were quite common. However, contemporary college students are in a time where the desire for materials and wealth is omnipresent and lures exist everywhere, thus the strengthening education is not so attractive to them and those social and practical educational activities are usually blocked and abandoned by a variety of factors. Based on this fact, it is necessary to find out new models or modes to ensure the effectiveness of the strengthening education. In order to improve the efficiency and effectiveness of modern college strengthening educational activity, traceless strengthening educational activity based on self-directed action mode is proposed by author. So-called traceless strengthening educational activity is a kind of strengthening educational activity difficult to detect, and the students are supposed to receive the educational content passively and unconsciously. So-called self-directed action mode is a way of action which must comply with self-directed principle, which is a rule that self-directed team members must decide for themselves and are responsible for their action. So-called self-directed team is a group formed for a particular purpose by self-directed action mode. The educators, as directors or superintendents, control the whole self-directed team and traceless strengthen educational activity based on self-directed mode. The educators have to know their prime duties, if they try for the good educators who are well-qualified for directors or superintendents in the traceless strengthening educational activity, as regards thinking and capability.

\section{Educators' Basic Responsibilities}

The educators of strengthening educational activities emerge as directors or superintendents in traceless strengthening educational activities based on self-directed action mode. It is the educators' basic responsibilities to administrate the whole traceless strengthening educational activities based on self-directed action mode. As viewed from managing traceless strengthening educational activities based on self-directed mode, the basic responsibilities of the educators are masterminding, organizing, decision-making, supervising, motivating and directing.

\section{A. Masterminding}

Commonly referred to as the primary management function of the educators, masterminding is the formulation of future courses of action. Masterminding is the action plan and the setting of educational purpose is given for a strengthening educational activity behind students' back. Masterminding and the objectives on which it is based limit purpose and direction to the self-directed team, its subunits, and contributing individuals, and the educators are the mastermind of the whole traceless strengthening educational activity based on self-directed action mode.

\section{B. Organizing}

So-called organizing is a process of integrating students, sources and conditions, in which both what the educators do is work out the best arrangement for carrying out its objectiverelated tasks, and the educational individuals and the educational resources are brought together for the purpose of attaining the traceless strengthening educational objectives. The arrangement of the tasks and persons in a traceless strengthening educational activities based on self-directed mode is one of the basic responsibilities of the educators, In fact, the educators should possess plentiful organizational 
experiences and skills if they want to be an effortless organizer. Therefore, as the good educators, they must have strong organizing ability in a traceless strengthening educational activity based on self-directed mode.

\section{Decision-making}

The general features of a traceless strengthening educational activity are passive, unconscious, uncertainty and volatility because this activity is a specific educational activity in which the students receive educational content and purpose passively and unconsciously. Therefore, a correct decisionmaking is the most important responsibilities of the educators in a traceless strengthening educational activities based on self-directed mode. Simply put, so-called decision-making denotes that a policy is formulated or a strategic decision is made. Making intelligent and ethical decisions is certainly a daunting challenge to the educators, because many traceless strengthening educational activities are complex, and potentially dangerous. And hence, the scientific analysis is usually very important for correct decision-making, though instincts and past decisions are a better guide to it. Generally, the educators should be familiar with the analysis process of the scientific decision-making to correct decision in practice as well as in theory, and often need to have better insights. In some cases, the scientific analysis is usually a complex process owing to some subjective and objective factors. Roughly speaking, the analysis process of the scientific decisionmaking is divided into four steps: (a) define objectives and collect information, (b) analyze information and formulate ideas, (c) evaluate ideas and select the best idea, (d) make implementing scheme and offer several alternatives.

\section{Supervising}

In fact, the educators not only have to deal with a series of affairs, such as masterminding, organizing, decisionmaking, doing ideological work and so on, but also must supervise the activities of self-directed teams themselves, if they want to obtain the anticipated effect or want to go well in traceless strengthening educational activities based on selfdirected action mode. So-called supervision is a practice that has to combine a good deal of experience with a certain amount of art, as vision and insight, and some science, particularly in the form of analysis and technique in traceless strengthening educational activities based on self-directed action mode. Generally speaking, the basic aspects of the educators' supervision are to administer self-directed teams' activities and to notice team members' safety. In most cases high quality traceless strengthening educational activities are based on "zero grievous mistake", "a sense of purpose" and so on. Therefore, the good educators should avoid going into too much loopholes and can use some ways to avoid widening them. Good self-directed teams need to have strong sense of purpose. Even arduous, maybe bitter, they can bear calmly. According to the traceless strengthen educational ideas, the educational purposes of the educators are hardly perceptible by the student, and the student is interpreted as a man of selfdependence who establishes his self-confident, and achieves his self-reliance and spiritual perfection by continuously selfdirecting actions in a traceless strengthen educational activity. While this happens, a traceless strengthen educational activity can be risky because it is an attempt to influence students using imperceptible educational way. So it is very important to pay attention to safe thought education of the students and the risk assessments of a traceless strengthen educational activity. If necessary, the educators may supervise the whole process of their activity to acquire maximum safety.

\section{E. Motivating}

Motivating students is an important aspect of the basic duties of the educators. When developing collage traceless strengthening education activity based on self-directed action mode we have to pay attention to motivating self-directed team. So-called motivating is that all the team based on self-directed action is inspired to pursue strengthening educational objectives. In other words, motivation means taking effective measures and actions so that team members are stimulated to finish their task as effectively as possible. One of the roles of the educators is to motivate the people who finish their task as effectively as possible in a self-directed traceless strengthening education activity. If team members are not motivated, they will not be interested in the educational activity they are doing. They will do slowly, be more likely to make mistakes and will not contribute to the educational activity. Many self-directed traceless strengthening education activities are especially tough and painstaking, so it is necessary that team members have the spirit of toughness and diligence. However, due to various reasons, many team members are the poor in hardworking and tough spirit. Directors should be good at motivating team members, and rouse them from indolence. If team members don't have a good attitude they won't do well. The educators' pep talk can help the confidence and morale of team members. The educators ought to keep active spirit attitude, because this can have a huge impact on team's morale. In fact, the inspiring educators can stimulate team members to succeed.

\section{F. Directing}

It is essential to offer the good directing in order to ensure to go well in the course of a traceless strengthening educational activity based on self-directed action mode. Socalled directing is a responsibility or process teaching, advising and guiding to students in a traceless strengthening educational activity based on self-directed action mode. Of course, it also means setting an example. On the one hand, directing is an important duty of the educators. This is because it's inevitable to educate and guide students in a traceless strengthening educational activity based on self-directed action mode, if the educators want to ensure that there is going well in the course of it. So directing is the basic responsibility of the educators. On the other hand, directing is a process teaching, advising and guiding to students. The educators must control the process of it, because they are managers of a traceless strengthening educational activity based on selfdirected action mode. From a certain angle, directing means 
that the activity shortfalls are blamed on the mentoring and not on the student. Therefore, any shortcoming identified in the activity will become the subject of the educating and guiding. In fact, a good directing to students' action is beneficial to ensure to go well in the course of a traceless strengthening educational activity based on self-directed action mode. Generally speaking, the directing is determined by the requirements of educational activity and students' safety. When a student is analyzed to show some capability for improvement, or the root cause of a fault is identified as a lack of knowledge, skill or experience on the part of him, the directing will be provided in time.

\section{Conclusion}

The traceless strengthening educational activity based on self-directed mode has many conspicuous advantages. Today, it is very significant to develop the traceless strengthening educational activity in university or college. At present, the outcomes are poor in traditional strengthen educational activities such as military training and morning exercises, because the manifestations of contradictious psychology are very outstanding in many young students. But in a traceless strengthening educational activity based on self-directed action mode, the educational purpose of constantly striving to strengthen oneself is hidden by a lively activity, and then there is a pretty good strengthen educational result to almost all young students including the young student with reverse psychology in the strengthening educational activities. The traceless strengthen educational activity based on self-directed mode can be to provide a lively and traceless atmosphere in the strengthening educational activity for students, and can make their enthusiasm of spontaneous acts run unprecedentedly high. In a traceless strengthening educational activity based on self-directed mode, the educators are being called upon to have strong power on masterminding, organizing, decision-making, supervising, motivating and directing. Several basic reasons are as follows; the first is traceless strengthening strategy and self-directed principle which are two main factors to cause the high security risks in traceless strengthening educational activities based on selfdirected action mode; the second is some human factors, such as lower awareness of the risks, severe loopholes of supervision and so on. In the whole traceless strengthening educational activity based on self-directed action mode, the educators function as the leading centre at all times. Therefore, the educators' basic responsibilities are vital, and it is highly necessary to discuss them.

\section{References}

[1] Shan Ying, The Original Theory of Higher Education. Beijing: Education and Science Press, 2008. (In Chinese)

[2] Sun Shaorong, Introduction to Higher Education Method. Shanghai: East China Normal University Publishing House, 2010. (In Chinese)

[3] Jiamei LU, Psychology and Education - Theory and Practice. Shanghai: Shanghai education Publishing House, 2011. (In Chinese)

[4] Gao Shenying, New Ideas for Effective Teaching - Studies of the Changes in Teaching of the Western Schools since the 1980s. Jinan: Shandong Education Press, 2011. (In Chinese)

[5] Hong YANG, An Exploration of Effect Evaluation Model for Limited Strengthening Education. In: Proceedings of 2012 International Symposium-Educational Research and Educational Technology. Melbourne: ST.PLUM-BLOSSOM PRESS, APR 2012. p335-339(In Australia)

[6] Hong YANG, An Exploration of a New Strengthening Education Model. International Conference on Computer Science and Education, 2012Vo1.5 No.1.

[7] Hong YANG, The Exploration and Research on the Decision Making Process and Organizing Work of the Traceless Strengthening Education of the College Students. In: The Conference on Management Innovation Intelligent Technology and Economic Development. Nanchang: Hong Kong Education and Research Press, July 2012. p198-202(In China). 